\title{
Binding Modes of Quinuclidinium Esters to Butyrylcholinesterase
}

\author{
Ines Primožič,* Tomica Hrenar, and Srđanka Tomić
}

Department of Chemistry, Faculty of Science, University of Zagreb, Horvatovac 102A, HR-10 000 Zagreb, Croatia

Abstract. The orientations of chiral quinuclidin-3-ol esters and benzoylcholine in the active site of horse butyrylcholinesterase have been investigated by flexible ligand docking. Change of the esters' acyl moiety as well as the substituent at the quinuclidinium nitrogen atom affected the activity and stereoselectivity of the biotransformations. Analysis of interactions in the active site revealed the most important binding patterns for enantiomers, which define their reactivity. Calculated Gibbs energies of binding obtained by molecular docking simulations were well correlated to the experimentally determined binding affinities of the investigated chiral esters. (doi: 10.5562/cca2060)

Keywords: butyrylcholinesterase, quinuclidinium esters, BChE-ester complexes, docking studies

\section{INTRODUCTION}

Butyrylcholinesterase (BChE, EC 3.1.1.8, also called cholinesterase, pseudocholinesterase or nonspecific cholinesterase) is a nonspecific ester hydrolyzing enzyme with function in cholinergic neurotransmission and probably involved in some other nervous system activities. ${ }^{1}$ It is present in nearly all body fluids and tissues in vertebrates and can hydrolyze various esters of choline and other compounds, as well as some organophosphorus compounds. Furthermore, BChE was used in the prophylaxis of hazardous organophosphorus poisoning (pesticides, insecticides and chemical warfare agents). ${ }^{2}$ It is believed that $\mathrm{BChE}$ is involved in the pathological development of Alzheimer's disease and $\mathrm{BChE}$ inhibitors have been used to raise acetylcholine levels and accordingly delay symptoms of the illness. ${ }^{3}$ Because of this and other significant pharmacological and toxicological functions it is important to understand and study interactions of small molecules in the active site of BChE.

The specific structure of the active site of cholinesterases has been revealed by the resolved crystal structures. ${ }^{4,5}$ Like in other serine hydrolases, BChE catalysed hydrolysis is carried out by a catalytic triad Ser200, Glu327 and His440 in the active centre of the protein. ${ }^{6}$ The imidazole ring of histidine transfers protons between serine and glutamate. Initially, an enzyme-substrate complex is formed. An ester acylenzyme intermediate is produced as a result of a nucleophilic attack of the serine hydroxyl group on the acyl group. Finally, a water molecule deacylates the enzyme (Scheme 1).

Derivatives of quinuclidin-3-ol are well known compounds with various pharmacological activities and are often the main or supportive part of the active substance. $^{7,8}$ In our previous work, ${ }^{9,10}$ chiral esters of quinuclidin-3-ol and benzoylcholine were subjected to enzymatic hydrolysis in the presence of $\mathrm{BChE}^{11,12}$ and the kinetics of BChE-catalyzed hydrolyses were determined. The hydrolysis of quaternary quinuclidine esters proved to be highly enantioselective. Kinetic studies have shown that $(R)$-enantiomers of all tested quinuclidinium esters are to a large extent better substrates of the enzyme than $(S)$-enantiomers. On the other hand, $(S)$-enantiomers showed higher affinity toward BChE (3-60 $\mu \mathrm{M})$. In order to explain experimental data con-

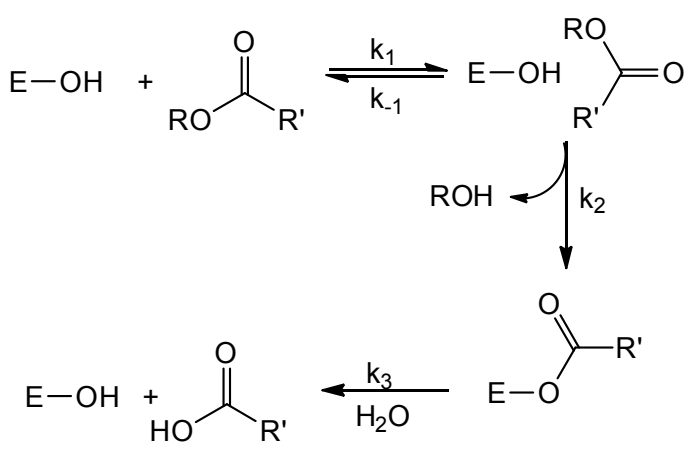

Scheme 1.

\footnotetext{
* Author to whom correspondence should be addressed. (E-mail: ines.primozic@chem.pmf.hr)
} 
cerning reactivity of enantiomers, we applied flexible ligand docking protocol, as implemented in AutoDock 4.2.2. ${ }^{13}$ To determine structural characteristics of Michaelis complexes, 11 compounds (Figure 1) were docked into the active site of the homology structural model of BChE. ${ }^{14}$

\section{EXPERIMENTAL}

The coordinates of $\mathrm{BChE}$ were obtained from homology modeling. ${ }^{14}$ The enzyme was prepared for docking using the Autodock Tools ${ }^{15}$ (ADT). Polar hydrogens were added and a Gasteiger partial charge was assigned to each atom.

Geometries of all ligands were optimized using the B3LYP functional ${ }^{16,17}$ and the 6-31G(d) basis set. All quantum chemical calculations were performed using the Gaussian 09 package. ${ }^{18}$ Non-polar hydrogens were removed from ligands and Gasteiger partial charges were assigned to all atoms using ADT. Torsional motion around single bonds was allowed (Figure 1).

Docking studies were performed by using the AutoDock 4.2.2 suite of programs. ${ }^{13,19}$ AutoDock requires a precalculated electrostatic grid map for each

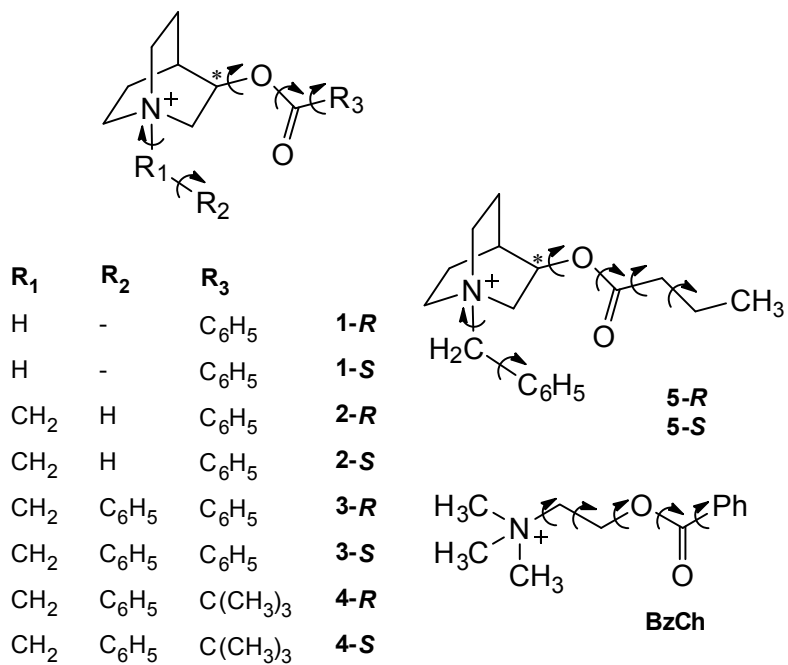

Figure 1. The ligands chosen for docking. Bonds where rotation was allowed are highlighted with arrows. $R$ and $S$ letters refer to the absolute configuration at position 3 of the quinuclidine ring.

atom type present in the substrate molecule. These electrostatic maps were calculated using the AutoGrid part of the suite with $0.2 \AA$ spacing between grid points and

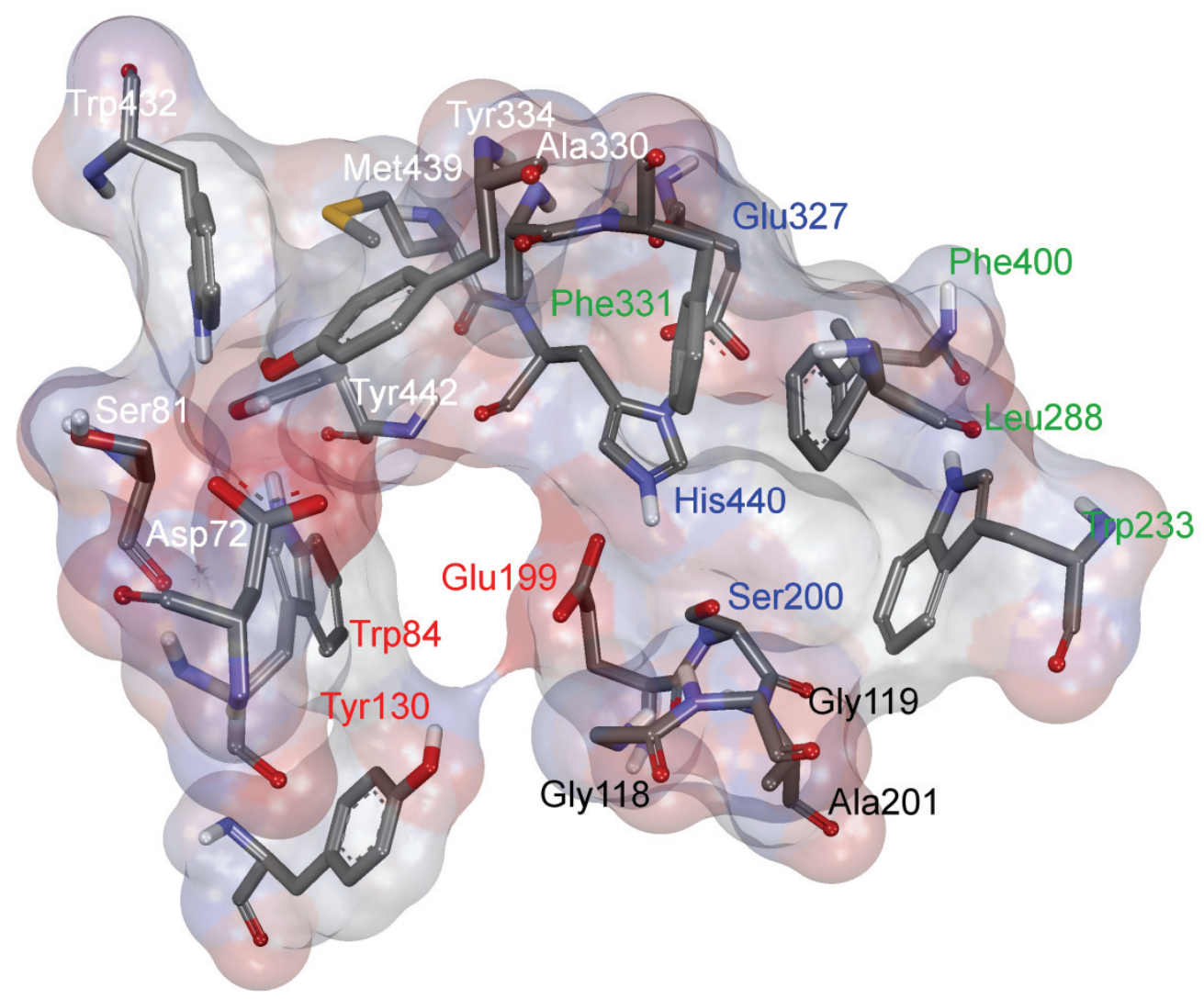

Figure 2. BChE active site major domains a) esteratic site (blue labels), b) acyl pocket (green labels), c) choline subsite (red labels), and d) the oxyanion hole (black labels). 
the centre of the grid was placed in the $\gamma$-oxygen atom of catalytic Ser200. Dimensions of the active site box were set to $20 \AA \times 20 \AA \times 20 \AA$ thus ensuring appropriate size of the ligand-accessible space. Consistencies of electrostatic maps were ascertained by checking maximum and minimum values of van der Waals energies and electrostatic potentials for each calculated grid map.

Flexible ligand docking was performed for 10 new compounds (5 enantiomeric pairs) as well as for benzoylcholine. Docking calculations were carried out using the Lamarckian genetic algorithm and all parameters were the same for each docking run. We used initially a population of 5000 random individuals, a maximum number of $2 \times 10^{7}$ energy evaluations, a maximum number of generations of $1 \times 10^{6}$, elitism value of 1 , mutation rate of 0.02 and crossover rate of 0.08 . For the local search, the pseudo-Solis and Wets method was used with a maximum of 10000 iterations per local search, the probability of performing a local search on an individual in the same population was 0.06, the maximum number of consecutive successes or failures before changing the size of local search space was 4 in both cases, initial size of local search space to sample was 1.0 and its lower bound was 0.01 . To ensure the validity of results, the docking procedure for each substrate consisted of 100 independent docking runs. The resulting positions were clustered according to an r.m.s.
Table 1. Experimentaly determined Michaelis $\left(K_{\mathrm{M}}\right)$ and inhibition $\left(K_{\mathrm{i}}\right)$ constants $^{8,10,12}$ along with Gibbs energies of binding predicted by AutoDock calculations $\left(t=30^{\circ} \mathrm{C}\right)$

\begin{tabular}{ccccc}
\hline Ester & $\frac{10^{3} \cdot K_{\mathrm{M}}}{\mathrm{mol} \mathrm{dm}^{-3}}$ & $\frac{10^{6} \cdot K_{\mathrm{i}}}{\mathrm{mol} \mathrm{dm}^{-3}}$ & $\frac{\Delta_{\mathrm{r}} G_{\mathrm{b}, \mathrm{exp}}^{\circ}}{\mathrm{kJ} \mathrm{mol}^{-1}}$ & $\frac{\Delta_{\mathrm{r}} G_{\mathrm{b}, \mathrm{calc}}^{\circ}}{\mathrm{kJ} \mathrm{mol}^{-1}}$ \\
\hline $\mathbf{1 - R}$ & 0.28 & - & $-20.62^{(\mathrm{a})}$ & -27.31 \\
$\mathbf{1 - S}$ & 0.13 & - & $-22.55^{(\mathrm{a})}$ & -33.82 \\
$\mathbf{2 - R}$ & 0.127 & - & $-22.61^{(\mathrm{a})}$ & -28.03 \\
$\mathbf{2 - S}$ & 0.065 & - & $-24.30^{(\mathrm{a})}$ & -31.82 \\
$\mathbf{3 - R}$ & 0.023 & - & $-26.92^{(\mathrm{a})}$ & -35.82 \\
$\mathbf{3 - S}$ & - & 3.3 & $-31.81^{(\mathrm{b})}$ & -40.88 \\
$\mathbf{4 - S}$ & - & 12.3 & $-28.50^{(\mathrm{b})}$ & -37.52 \\
$\mathbf{5 - S}$ & - & 60.5 & $-24.48^{(\mathrm{b})}$ & -35.44 \\
\hline
\end{tabular}

(a) Estimated as $\Delta_{\mathrm{r}} G_{\mathrm{b}}^{\circ} \approx-R T \ln K_{\mathrm{M}}{ }^{-1}$.

(b) Calculated as $\Delta_{\mathrm{r}} G_{\mathrm{b}}^{\circ}=-R T \ln K_{\mathrm{i}}^{-1}$.

criterion of $0.5 \AA$. Obtained structures were analyzed visually and evaluated based on their interactions with the amino acids within the active site of the enzyme.

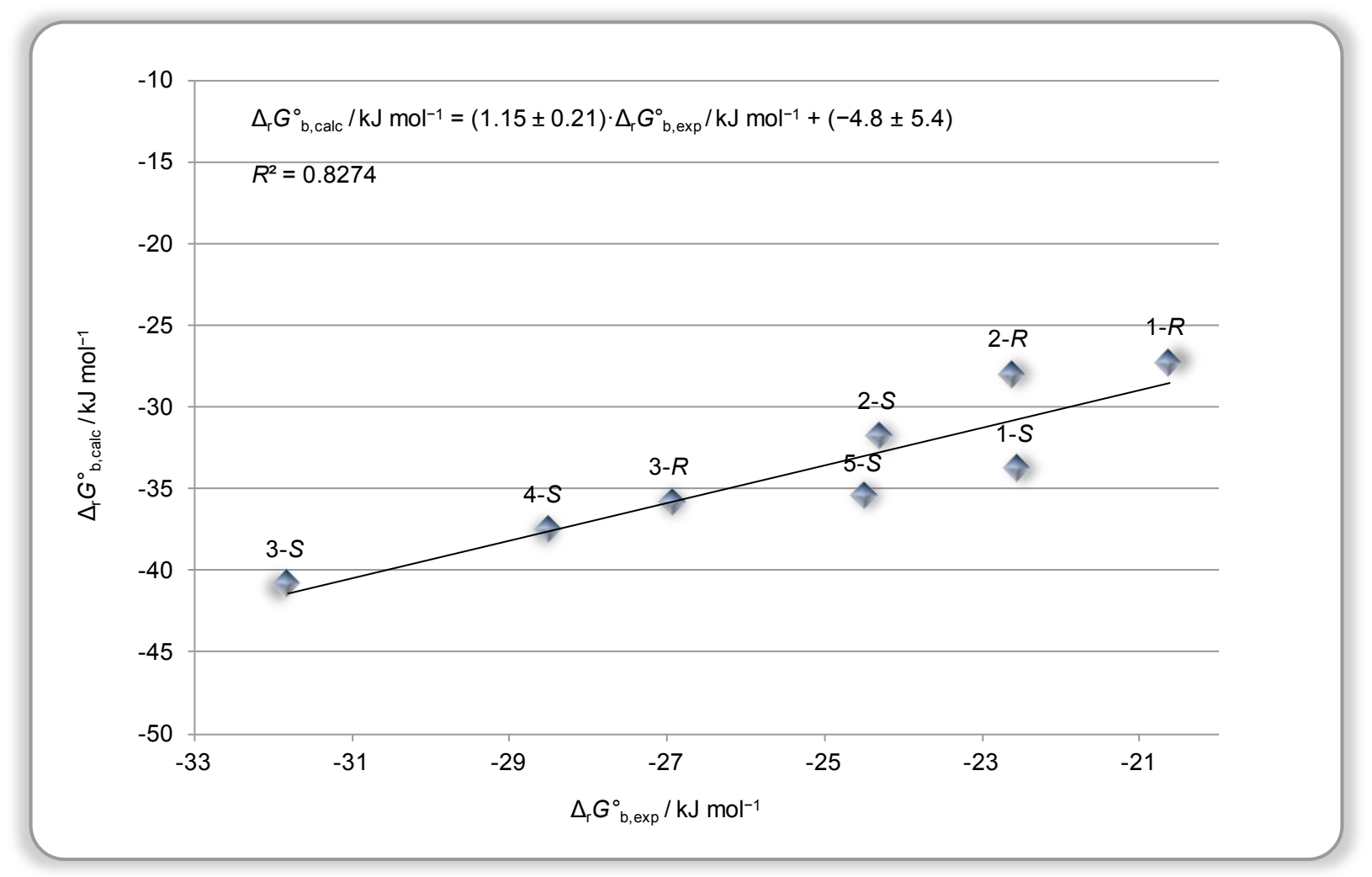

Figure 3. Gibbs energies of binding $\Delta_{\mathrm{r}} G_{\mathrm{b}, \mathrm{calc}}^{\circ}$ calculated by AutoDock compared to the experimentally determined Gibbs energies $\Delta_{\mathrm{r}} G_{\mathrm{b}, \mathrm{exp}}^{\circ}$. 

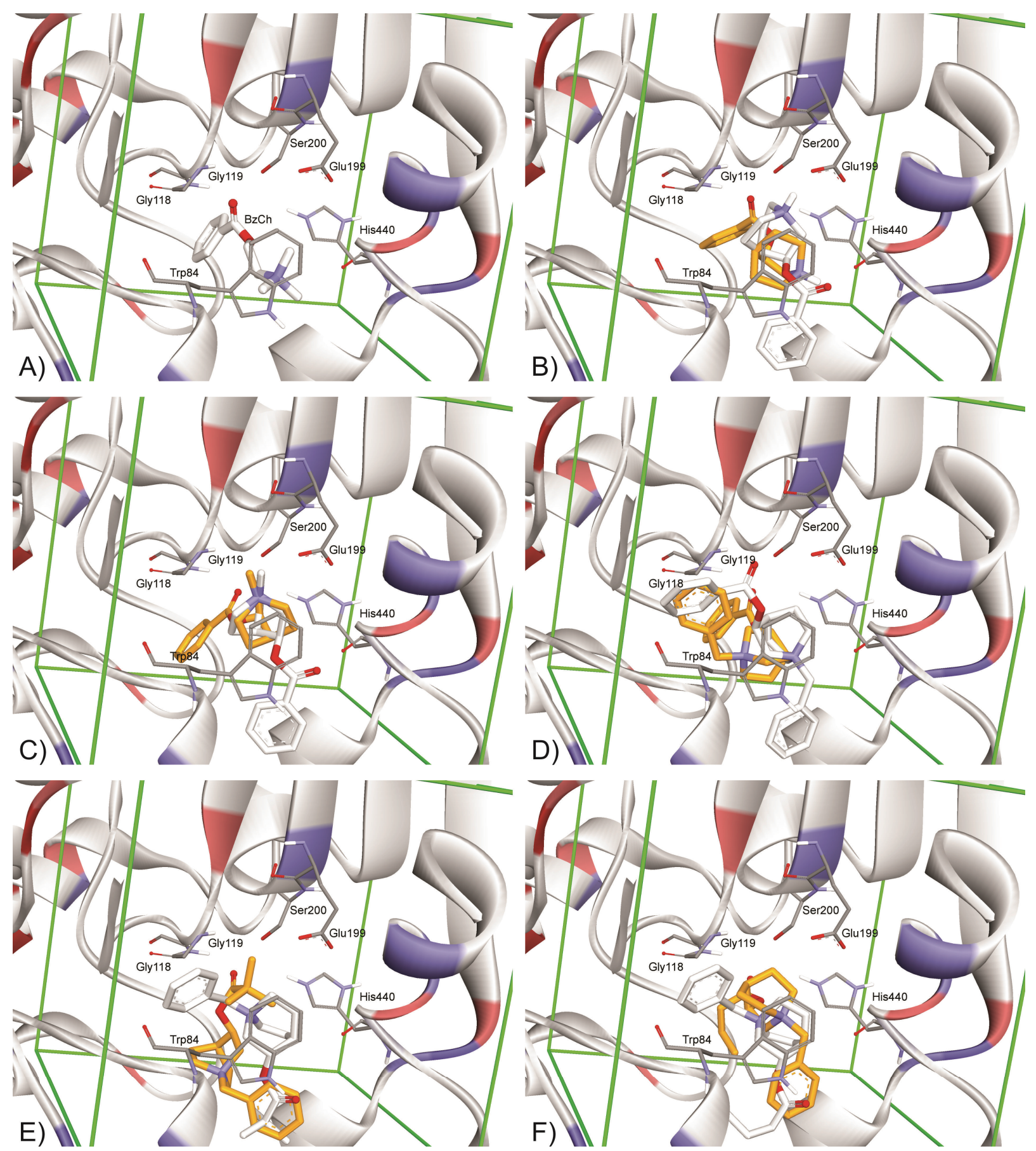

Figure 4. Substrate-BChE complexes derived from docking study. BChE active site is represented by some structurally important amino acids. Nonpolar hydrogen atoms are omitted for clarity. The entrance to the active site gorge is from below. $(S)$-enantiomer (carbon atoms white), $(R$ )-enantiomer (carbon atoms orange): A) BzCh, B) 1; C) 2; D) 3; E) 4; F) 5. Selected interatomic distances are given in Table 2 .

\section{RESULTS AND DISCUSSION}

In order to explain and rationalize experimentally obtained data, enantiomers of five different quinuclidinium esters (1-5) and benzoylcholine (BzCh) were docked into the active site of BChE by utilizing the AutoDock program. The $\mathrm{BChE}$ active site gorge ${ }^{14}$
(Figure 2) consists of several major regions: a) esteratic site containing the active serine as a part of catalytic triad (Ser, His, Glu), b) acyl pocket - a hydrophobic region which accommodates the acyl group of an ester, c) choline subsite for the recognition of the substrate's quaternary ammonium group and d) the oxyanion hole formed by the main chain 
Table 2. Selected interatomic distances between ligands and some important amino acids within the BChE active site (values not shown are greater than $8 \AA$ )

\begin{tabular}{|c|c|c|c|c|}
\hline Comp. & $\frac{r\left(\mathrm{~N}-\mathrm{N}_{\text {Trp84 }}\right)^{(\mathrm{a})}}{\AA}$ & $\frac{r\left(\mathrm{C}-\mathrm{O}_{\text {Ser200 }}\right)^{(\mathrm{b})}}{\AA}$ & $\frac{r\left(\mathrm{O}-\mathrm{N}_{\mathrm{Gly} \mid 18}\right)^{(\mathrm{c})}}{\AA}$ & $\frac{r\left(\mathrm{O}-\mathrm{N}_{\mathrm{Gly} 119}\right)^{(\mathrm{c})}}{\AA}$ \\
\hline $\mathrm{BzCh}$ & 4.570 & 3.249 & 3.183 & 2.631 \\
\hline $1-R$ & 4.722 & 3.005 & 2.796 & 3.095 \\
\hline $1-S$ & 6.159 & - & - & - \\
\hline $2-R$ & 5.689 & 3.671 & 3.720 & 2.951 \\
\hline $2-S$ & 5.766 & - & - & - \\
\hline $3-R$ & 4.752 & 3.434 & 3.466 & 3.111 \\
\hline $3-S$ & 3.948 & 6.228 & 3.667 & 6.032 \\
\hline $4-R$ & 5.770 & 3.794 & 3.077 & 2.881 \\
\hline $4-S$ & 4.977 & - & - & - \\
\hline $5-R$ & 4.704 & 3.492 & 4.550 & 3.166 \\
\hline $5-S$ & 4.955 & - & - & - \\
\hline
\end{tabular}

(a) From the ligand's quaternary $\mathrm{N}$ atom to the side chain Trp84 indole nitrogen atom.

(b) From the ligand's carbonyl C atom to the side chain Ser200 $\gamma$-oxygen atom.

(c) From the ligand's carbonyl $\mathrm{O}$ atom to the backbone Gly nitrogen atom.

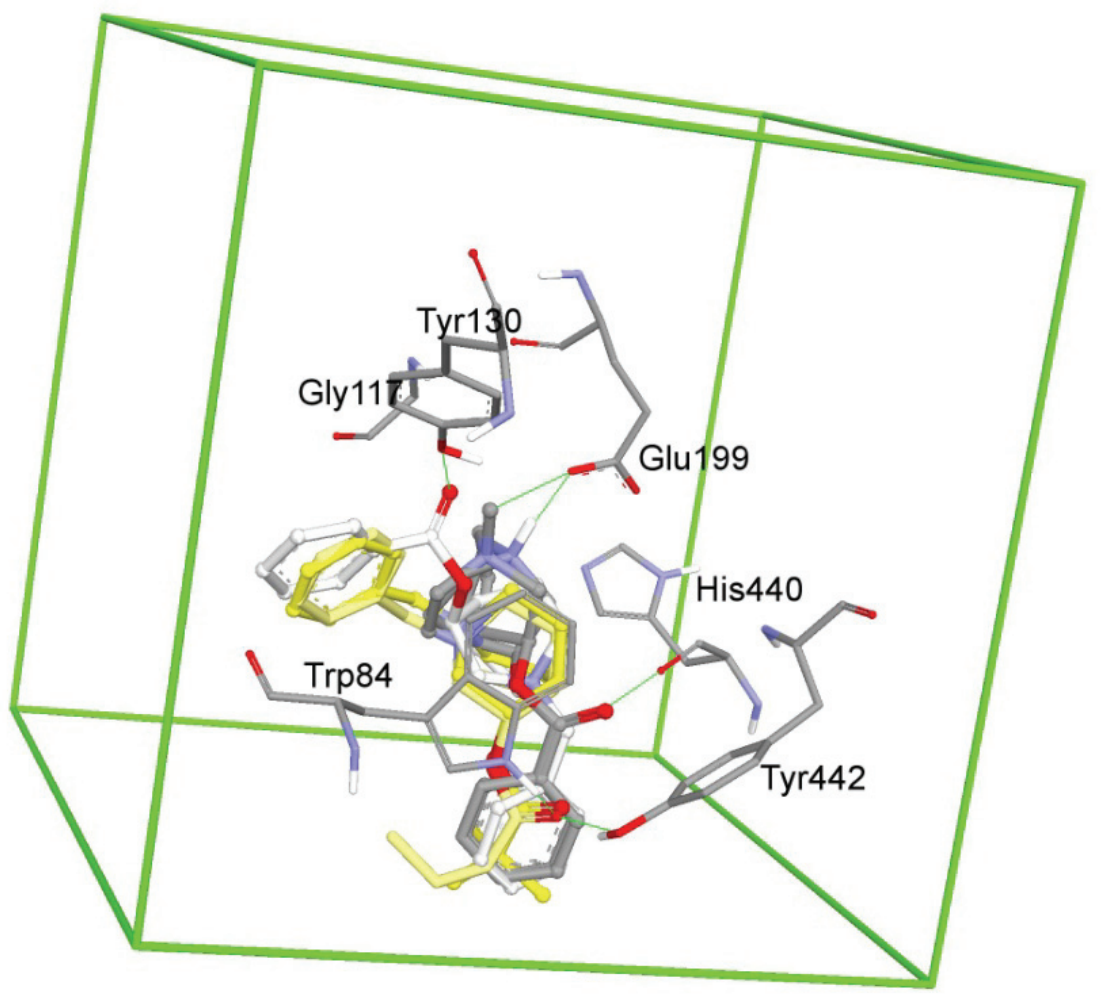

Figure 5. Superimposed geometries of (S)-enantiomers: grey stick model (1), grey ball and stick model (2), white ball and stick model (3), yellow ball and stick model (4) and pale yellow stick model (5) obtained by docking simulations. Only amino acids having a close contact with the substrate's carboxyl oxygen atoms are presented (green lines). Nonpolar hydrogen atoms are omitted for clarity. 
$\mathrm{N}-\mathrm{H}$ dipoles interacting with the carbonyl oxygen atom of the substrate.

Several clusters of binding orientations for all substrates were obtained. Since $(S)$-enantiomers were much better inhibitors than the substrates of the enzyme, clusters with the lowest absolute energy values for $(S)$ enantiomers were compared with the energies of the correctly placed geometries of $(R)$-enantiomers (rather good substrates) for the reaction to occur. The correct position for hydrolysis to occur was assumed to be the one with the acyl moiety in the acyl pocket and the ester carboxyl group in the vicinity of catalytic Ser hydroxyl group. In Table 1, the binding energies predicted by AutoDock in conjunction with the kinetic data compiled from the literature are shown.

The calculated docking energies for quinuclidinium esters were well correlated to the experimentally obtained values $\left(R^{2}=0.8274\right)$ as shown in Figure 3. Even better correlation is found within the series of only $(R)$ enantiomers of compounds $\mathbf{1}-\mathbf{3}\left(R^{2}=0.9445\right)$ or only $(S)$ enantiomers of compounds $\mathbf{1}-\mathbf{5}\left(R^{2}=0.8314\right)$. The good quantitative agreement between computed and experimental values confirmed the correctness of the chosen docking method and analyses applied.

Substrate-BChE complexes derived from docking studies for quinuclidinium compounds 1-5 and benzoylcholine are shown in Figure 4. Analyses of the docked geometries for each pair of enantiomers revealed trends in binding associated with the stereochemistry of substrates. $(R)$-enantiomers of all compounds and benzoylcholine are bound productively: the carbonyl group is found in the vicinity of hydroxyl oxygen atom of Ser200, forming two H-bonds with the backbone Gly118 and Gly119 amide group (Table 2). The quaternary quinuclidinium group of all enantiomers is positioned in the choline binding site and its orientation depends on the bulkiness of the substituents at the quinuclidinium nitrogen atom. Thus, compounds $\mathbf{1}$ and $\mathbf{2}$ with the smallest substituents (protonated and methyl substituted $(S)$-quinuclidinium derivatives respectively) are bound in a similar way and are positioned in the vicinity of the Glu199 side chain: (a strong H-bond of 1$\boldsymbol{S}(2.6 \AA)$ and $\mathbf{2 - S}(2.8 \AA))$. On the other hand, $N$-benzyl substituted compounds cannot have similar stabilization due to the present bulky aromatic moiety. Therefore, two different modes of binding for $(S)$ - $N$-benzyl enantiomers are found - one for the esters of aliphatic acids (pivalic 4 and butyric 5) and a different one for the best inhibitor, $(S$ )-ester of benzoic acid 3 (Figure 5). In 4-S and $\mathbf{5}-\boldsymbol{S}$ complexes with $\mathrm{BChE}$, carboxyl oxygen atom is pointing away from the catalytic triad and establishes strong H-bonds with the Tyr442 side chain hydroxyl group and the Trp84 indole amino group (2.9 $\AA)$. The benzyl moiety is positioned in the hydrophobic region near the $\operatorname{Trp} 84$ backbone and in the vicinity of the
Tyr130 side chain. The aromatic ring of the acyl part of $\mathbf{3 - S}$ is also stabilized in this domain. The carboxyl group of 3-S has two H-bonds as well: the Tyr130 side chain hydroxyl group $(2.7 \AA)$ and the Gly117 backbone amide $(3.3 \AA)$. The additional favourable interactions of two aromatic parts of $\mathbf{3}-\boldsymbol{S}$ may be the reason for the best inhibitory power of this compound. These results supported our previous findings based on experimental data and quantum-chemical calculations ${ }^{11,12}$ that $(S)$ enantiomers compared to $(R)$ - have stronger interactions within the active site of BChE which successfully compete with productive binding leading to the tetrahedral intermediate and subsequent hydrolysis.

\section{CONCLUSION}

Differences, similarities and relations among enantiomers of selected quinuclidinium esters were studied with molecular docking simulations in the active site of $\mathrm{BChE}$ to determine the origins of observed activity and stereoselectivity of the enzyme. The calculated docking energies were well correlated with the experimental values and supported the experimental data for binding affinities of chiral quinuclidinium esters. This study demonstrated that flexible ligand docking is appropriate and useful tool for studying $\mathrm{BChE}$ binding specificity of quinuclidinium compounds.

Acknowledgements. We wish to thank Professor Israel Silman of the Weizmann Institute, Rehovot, Israel for providing coordinates of the theoretical structure of human BChE. This work was supported by the Croatian Ministry of Science, Education and Sports, Research Project No. 119-1191344-3121.

\section{REFERENCES}

1. S. Darvesh, D. A. Hopkins, and C. Geula, Nature Rev. Neurosci. 4 (2003) 131-138.

2. J. Bajgar, J. Med. Chem. Def. 1 (2004) 1-16.

3. E. Giacobini, Drugs Aging 18 (2001) 891-898.

4. Y. Nicolet, O. Lockridge, P. Masson, J. C. Fontecilla-Camps, and F. Nachon, J. Biol. Chem. 278 (2003) 41141-41147.

5. J. P. Colletier, D. Fournier, H. M. Greenblatt, J. Stojan, J. L. Sussman, G. Zaccai, I. Silman, and M. Weik, EMBO J. 25 (2006) 2746-2756.

6. P. Taylor and Z. Radić, Annu. Rev. Pharmacol. Toxicol. 34 (1994) 281-320

7. M. D. Mashkovsky, L. N. Yakhontov, M. E. Kaminka, and E. E. Mikhlina, Prog. Drug Res. 27 (1983) 9-61.

8. I. Primožič, T. Hrenar, S. Tomić, and Z. Meić J. Phys. Org. Chem. 58 (2002) 608-614.

9. I. Primožič, M. Bolant, A. Ramić, and S. Tomić, Molecules $\mathbf{1 7}$ (2012) 786-795.

10. I. Primožič and S. Tomić, Croat. Chem. Acta 84 (2011) 245-249.

11. I. Primožič, T. Hrenar, S. Tomić, and Z. Meić, Croat. Chem. Acta 76 (2003) 93-99.

12. I. Primožič, T. Hrenar, S. Tomić, and Z. Meić, Eur. J. Org. Chem. (2003) 295-301. 
13. G. M. Morris, D. S. Goodsell, R. S. Halliday, R. Huey, W. E. Hart, R. K. Belew, and A. J. Olson, J. Comput. Chem. 19 (1998) 1639-1662.

14. M. Harel, J. L. Sussman, E. Krejci, S. Bon, P. Chanal, J. Massoulie, and I. Silman, Proc. Natl. Acad. Sci. USA 89 (1992) 10827-10831.

15. M. F. Sanner, J. Mol. Graphics Mod. 17 (1999) 57-61.

16. A. D. Becke, J. Chem. Phys. 98 (1993) 5648-5652.

17. C. Lee, W.Yang, and R. G. Parr, Phys. Rev. B 37 (1988) 785 789.

18. Gaussian 09, Revision A.02, M. J. Frisch, G. W. Trucks, H. B. Schlegel, G. E. Scuseria, M. A. Robb, J. R. Cheeseman, G. Scalmani, V. Barone, B. Mennucci, G. A. Petersson, H. Nakatsuji, M. Caricato, X. Li, H. P. Hratchian, A. F. Izmaylov, J. Bloino, G. Zheng, J. L. Sonnenberg, M. Hada, M. Ehara, K.
Toyota, R. Fukuda, J. Hasegawa, M. Ishida, T. Nakajima, Y. Honda, O. Kitao, H. Nakai, T. Vreven, J. A. Montgomery, Jr., J. E. Peralta, F. Ogliaro, M. Bearpark, J. J. Heyd, E. Brothers, K. N. Kudin, V. N. Staroverov, R. Kobayashi, J. Normand, K. Raghavachari, A. Rendell, J. C. Burant, S. S. Iyengar, J. Tomasi, M. Cossi, N. Rega, J. M. Millam, M. Klene, J. E. Knox, J. B. Cross, V. Bakken, C. Adamo, J. Jaramillo, R. Gomperts, R. E. Stratmann, O. Yazyev, A. J. Austin, R. Cammi, C. Pomelli, J. W. Ochterski, R. L. Martin, K. Morokuma, V. G. Zakrzewski, G. A. Voth, P. Salvador, J. J. Dannenberg, S. Dapprich, A. D. Daniels, O. Farkas, J. B. Foresman, J. V. Ortiz, J. Cioslowski, and D. J. Fox, Gaussian, Inc., Wallingford CT, 2009.

19. R. Huey, G. M. Morris, A. J. Olson, and D. S. Goodsell, J. Comput. Chem. 28 (2007) 1145-1152. 